Yang Liguo*, Zhang Chaoyang, Wang Kai, Zhai Shengxian and Liu Nana

\title{
The crystal structure of bis $\left(\mu_{2}-5\right.$-chloro- $N-(2-$ methyl-1-oxidopropylidene)-2- oxidobenzohydrazonate- $\left.\kappa^{5} N, O, O^{\prime}: N^{\prime}, O^{\prime \prime}\right)$ pentakis(pyridine- $\kappa^{1} N$ )tricopper(II), $\mathrm{C}_{47} \mathrm{H}_{45} \mathrm{Cl}_{2} \mathrm{~N}_{9} \mathrm{Cu}_{3} \mathrm{O}_{6}$
}

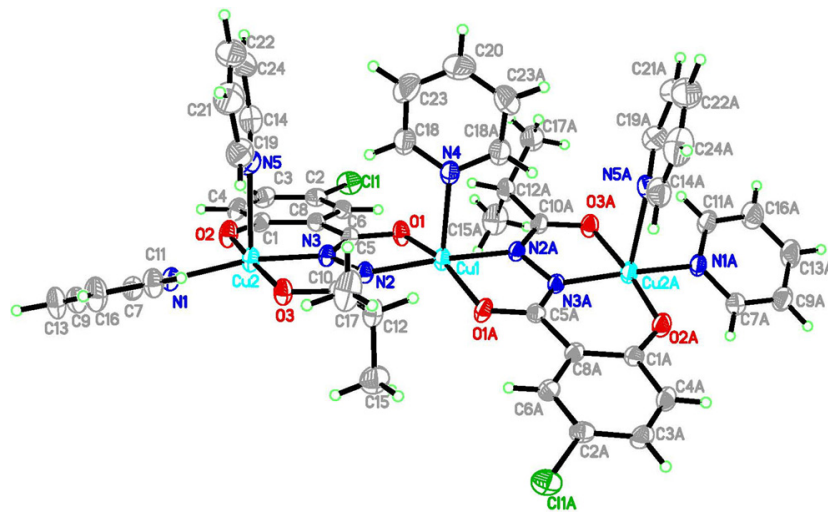

https://doi.org/10.1515/ncrs-2020-0601

Received November 16, 2020; accepted January 22, 2021; published online February 12, 2021

\begin{abstract}
$\mathrm{C}_{47} \mathrm{H}_{45} \mathrm{Cl}_{2} \mathrm{~N}_{9} \mathrm{Cu}_{3} \mathrm{O}_{6}$, monoclinic, $\mathrm{C} 2 / \mathrm{c}$ (no. 15), $a=24.084(9) \AA, \quad b=12.152(5) \AA, c=17.404(7) \AA$, $\beta=109.984(4)^{\circ}, V=4787(3) \AA^{3}, Z=4, R_{g t}(F)=0.0455$, $w R_{\text {ref }}\left(F^{2}\right)=0.1363, \mathrm{~T}=298 \mathrm{~K}$.
\end{abstract}

\section{CCDC no.: 2057941}

The molecular structure is shown in the Figure. Table 1 contains crystallographic data and Table 2 contains the list of the atoms including atomic coordinates and displacement parameters.

*Corresponding author: Yang Liguo, College of Chemistry and Environmental Engineering, Anyang Institute of Technology, Anyang 455000, Henan, P. R. China, E-mail: Igyang@ayit.edu.cn. https:// orcid.org/0000-0003-4899-8298

Zhang Chaoyang, Wang Kai, Zhai Shengxian and Liu Nana, College of Chemistry and Environmental Engineering, Anyang Institute of Technology, Anyang 455000, Henan, P. R. China. https://orcid.org/ 0000-0002-2016-1034 (Z. Shengxian)
Table 1: Data collection and handling.

\begin{tabular}{ll}
\hline Crystal: & Yellow block \\
Size: & $0.08 \times 0.06 \times 0.03 \mathrm{~mm}$ \\
Wavelength: & Mo $K \alpha$ radiation $(0.71073 \AA$ $)$ \\
$\mu:$ & $1.49 \mathrm{~mm}^{-1}$ \\
Diffractometer, scan mode: & Bruker APEX-II, $\varphi$ and $\omega$ \\
$\theta_{\text {max }}$, completeness: & $25.0^{\circ},>99 \%$ \\
$N\left(h k l_{\text {measured }}, N(h k l)_{\text {unique }}, R_{\text {int }}:\right.$ & $26,523,4198,0.085$ \\
Criterion for $I_{\text {obs }}, N(h k l)_{\text {gt }}:$ & $I_{\text {obs }}>2 \sigma\left(I_{\text {obs }}\right), 3394$ \\
$N(\text { param })_{\text {refined }}:$ & 306 \\
Programs: & Bruker $[1]$, SHELX $[2,3]$ \\
\hline
\end{tabular}

\section{Source of material}

The $N$-(5-chloro-acetylsalicyloylhydrazine) was synthesized according to reference [4]. A mixture of $\mathrm{N}$-(5-chloro-acetylsalicyloylhydrazine) (0.196 g, $1 \mathrm{mmol})$ and acetate copper $(0.186 \mathrm{~g}, 1 \mathrm{mmol})$ in $10 \mathrm{ml}$ methanol was stirred at room temperature for $1 \mathrm{~h}$. The mixture was filtered to remove impurties, and then left at room temperature. After a few days, yellow block crystals had formed. Yield (0.13 g, 43\%).

\section{Experimental details}

The $\mathrm{H}$ atoms were geometrically placed $(\mathrm{C}-\mathrm{H}=0.95-0.98 \AA$ ) and refined as riding with $U_{\text {iso }}(\mathrm{H})=1.2-1.5 U_{\text {eq }}(\mathrm{C})$. The N-bound $\mathrm{H}$ atoms were located in a difference Fourier map but were refined with a distance restraint of $\mathrm{N}-\mathrm{H}=0.88 \pm 0.01 \AA$, and with $U_{\text {iso }}(\mathrm{H})$ set to $1.2 U_{\text {eq }}(\mathrm{N})[1-3]$.

\section{Comment}

Multidenate ligands have been synthesized in recent years for the polynuclear metal complexes [4, 5]. The $\mathrm{N}$ atoms and $\mathrm{O}$ atoms can coordinated with the transition metals and rare 
Table 2: Fractional atomic coordinates and isotropic or equivalent isotropic displacement parameters $\left(\AA^{2}\right)$.

\begin{tabular}{|c|c|c|c|c|}
\hline Atom & $x$ & $y$ & $z$ & $U_{\text {iso }} * / U_{\text {eq }}$ \\
\hline Cu1 & 0.5000 & $0.79108(5)$ & 0.2500 & $0.03580(18)$ \\
\hline Cu2 & $0.36073(2)$ & $0.80720(4)$ & $0.37855(2)$ & 0.04015 (17) \\
\hline $\mathrm{Cl} 1$ & $0.65323(4)$ & $0.92636(9)$ & $0.66705(6)$ & 0.0608 (3) \\
\hline 01 & $0.52885(10)$ & $0.8181(2)$ & $0.36925(14)$ & $0.0441(6)$ \\
\hline 02 & $0.40102(10)$ & $0.8365(2)$ & $0.49188(14)$ & $0.0500(6)$ \\
\hline 03 & $0.32620(10)$ & $0.7873(2)$ & $0.25903(14)$ & $0.0449(6)$ \\
\hline N1 & $0.28051(12)$ & $0.8209(2)$ & 0.39057 (17) & $0.0404(6)$ \\
\hline $\mathrm{N} 2$ & $0.42327(11)$ & $0.7972(2)$ & 0.26899 (16) & $0.0331(6)$ \\
\hline N3 & $0.43176(11)$ & $0.8152(2)$ & $0.35292(16)$ & $0.0338(6)$ \\
\hline N4 & 0.5000 & $0.5938(4)$ & 0.2500 & $0.0482(10)$ \\
\hline N5 & $0.36530(15)$ & $0.5991(3)$ & $0.3945(2)$ & 0.0564 (8) \\
\hline C1 & $0.45822(14)$ & $0.8552(3)$ & 0.52629 (19) & $0.0390(7)$ \\
\hline C2 & 0.57809 (15) & $0.8991(3)$ & $0.6137(2)$ & $0.0431(8)$ \\
\hline C3 & $0.53756(17)$ & $0.9051(3)$ & $0.6534(2)$ & $0.0487(9)$ \\
\hline H1 & 0.5495 & 0.9234 & 0.7086 & $0.058^{\star}$ \\
\hline C4 & $0.47916(16)$ & $0.8836(3)$ & $0.6103(2)$ & $0.0482(9)$ \\
\hline $\mathrm{H} 2$ & 0.4521 & 0.8880 & 0.6376 & $0.058^{\star}$ \\
\hline C5 & 0.48689 (13) & $0.8262(3)$ & 0.39902 (19) & $0.0346(7)$ \\
\hline C6 & $0.56057(14)$ & $0.8730(3)$ & 0.53209 (19) & $0.0399(7)$ \\
\hline H3 & 0.5885 & 0.8703 & 0.5062 & $0.048^{\star}$ \\
\hline $\mathrm{C} 7$ & 27292 (16) & $0.8784(3)$ & $0.4520(2)$ & $.0497(9)$ \\
\hline $\mathrm{H} 4$ & 0.3052 & 0.9145 & 0.4886 & $0.060^{*}$ \\
\hline $\mathrm{C} 8$ & $0.50107(14)$ & $0.8504(3)$ & 0.48715 (19) & $0.0354(7)$ \\
\hline C9 & 0.21897 (18) & $0.8858(4)$ & $0.4627(3)$ & $0.0584(10)$ \\
\hline H5 & 0.2152 & 0.9264 & 0.5059 & $0.070^{\star}$ \\
\hline C10 & $0.36667(14)$ & $0.7839(3)$ & $2260(2)$ & $0.0375(7)$ \\
\hline C11 & $0.23307(16)$ & $0.7723(4)$ & $0.3378(2)$ & $0.0538(10)$ \\
\hline H6 & 0.2373 & 0.7346 & 0.2936 & $0.065^{*}$ \\
\hline C12 & $0.34836(15)$ & $0.7644(4)$ & $0.1358(2)$ & $0.0497(9)$ \\
\hline $\mathrm{H} 7$ & 0.3831 & 0.7407 & 1230 & $0.060^{*}$ \\
\hline C13 & 0.17097 (18) & $.8331(4)$ & .4095 (3) & $0.0696(12)$ \\
\hline $\mathrm{H} 8$ & 0.1343 & 360 & 0.4163 & $0.084^{\star}$ \\
\hline C14 & $0.4039(2)$ & $0.5553(4)$ & $4601(3)$ & $0.0705(12)$ \\
\hline H9 & 0.4325 & 0.6008 & 0.4952 & $0.085^{*}$ \\
\hline C15 & $3264(2)$ & $.8737(5)$ & $.0916(3)$ & $0.0791(14)$ \\
\hline $\mathrm{H} 10 \mathrm{~A}$ & 0.2929 & 0.8990 & 0.1046 & $0.119^{\star}$ \\
\hline $\mathrm{H} 10 \mathrm{~B}$ & 0.3151 & 0.8629 & 0.0336 & $0.119^{\star}$ \\
\hline $\mathrm{H} 10 \mathrm{C}$ & 0.3574 & 0.9274 & .1089 & $0.119^{\star}$ \\
\hline C16 & .17845 (18) & $0.7755(5)$ & $0.3456(3)$ & $0.0721(13)$ \\
\hline H11 & 0.1467 & 0.7391 & 0.3081 & $0.087^{*}$ \\
\hline C17 & $0.3023(2)$ & $6751(5)$ & 1093 (3) & $0.0823(16)$ \\
\hline $\mathrm{H} 12 \mathrm{D}$ & 0.3179 & 0.6083 & 0.1380 & $0.123^{*}$ \\
\hline $\mathrm{H} 12 \mathrm{G}$ & 0.2917 & 0.6631 & 0.0515 & $0.123^{*}$ \\
\hline H12J & 0.2679 & 0.6970 & 0.1215 & $0.123^{*}$ \\
\hline C18 & $0.4724(2)$ & $0.5355(4)$ & 0.2907 (3) & $0.0695(12)$ \\
\hline H13 & 0.4521 & 0.5736 & .3192 & $0.083^{*}$ \\
\hline C19 & $3256(2)$ & $.5334(4)$ & $.3451(3)$ & $0.0697(12)$ \\
\hline H14 & 0.2993 & 0.5636 & 0.2975 & $0.084^{\star}$ \\
\hline $\mathrm{C} 20$ & 0.5000 & $0.3652(6)$ & 0.2500 & $0.089(2)$ \\
\hline H15 & 0.5000 & 0.2887 & 0.2500 & $0.107^{\star}$ \\
\hline $\mathrm{C} 21$ & $0.3205(3)$ & $0.4237(5)$ & $0.3587(4)$ & 0.0900 (17) \\
\hline H16 & 0.2905 & 0.3815 & 0.3228 & $0.108^{\star}$ \\
\hline$C 22$ & $0.3599(4)$ & $0.3789(5)$ & $0.4253(5)$ & $0.106(2)$ \\
\hline H17 & 0.3581 & 0.3042 & 0.4357 & $0.127^{\star}$ \\
\hline $\mathrm{C} 23$ & $0.4720(3)$ & $0.4230(4)$ & 2932 (4) & 884 (16) \\
\hline
\end{tabular}

Table 2: (continued)

\begin{tabular}{lrrrr}
\hline Atom & $\boldsymbol{x}$ & $\boldsymbol{y}$ & $\boldsymbol{z}$ & $\boldsymbol{U}_{\text {iso }} \boldsymbol{U}_{\text {eq }}$ \\
\hline $\mathrm{H} 28$ & 0.4529 & 0.3864 & 0.3239 & $0.106^{*}$ \\
$\mathrm{C} 24$ & $0.4043(3)$ & $0.4446(6)$ & $0.4797(4)$ & $0.0967(19)$ \\
$\mathrm{H} 19$ & 0.4323 & 0.4153 & 0.5263 & $0.116^{*}$ \\
\hline
\end{tabular}

earth metals, so lots of the polynuclear metal complexes can be producted. The magnetic properties of the polynuclear metal complexes have been studied, which have good magnetic properties [6-8]. So lots of the polynuclear metal complexes were producted [9-10]. In this paper, one new $\mathrm{Cu}$ is described and the structure is similar to reference [8].

The title compound crystallizes in the monoclinic space group $C 2 / c$ with one half of the complex in the asymmetric unit. The bond lengths of $\mathrm{Cu}-\mathrm{O}$ are 1.978, 1.911 and $1.974 \AA$, the bond lengths of $\mathrm{Cu}-\mathrm{N}$ are 1.987, 2.397, 1.912 and $2.020 \AA$, respectively. The bond lengths of $\mathrm{Cu}-\mathrm{N}$ from the pyridine ligands are longer than the other $\mathrm{Cu}-\mathrm{N}$ bond lengths, which are similar with the reference [8]. Each copper coordination environment in the complex exhibits a distorted pyramidal geometry. The trinuclear complex is centrosymmetric.

Author contributions: All the authors have accepted responsibility for the entire content of this submitted manuscript and approved submission.

Research funding: Natural Science Foundation of Henan Province of China (No. 202300410010), Research Fund of Anyang Institute of Technology (Nos. YPY2019011, YPY2020025), Key discipline of environmental engineer of Anyang Institute of Technology.

Conflict of interest statement: The authors declare no conflicts of interest regarding this article.

\section{References}

1. Bruker. SMART and SAINT for Windows NT Software Reference Manuals, Version 5.0; Bruker Analytical X-Ray Systems: Madison, WI, 1997.

2. Sheldrick G. M. SADABS, A Software for Empirical Absorption Correction; University of Göttingen: Göttingen: Germany, 1997.

3. SHELXL Reference Manual, Version 5.1. Bruker Analytical X-Ray Systems, Madison, WI, 1997.

4. Yang M. X., Lin S., Chen L. J., Liu S. X. Synthesis and crystal structure of the trinuclear nickel(II) complex with Schiff base ligand N-butylsalicylhydrazide. Chin. J. Inorg. Chem. 2003, 19, 434-436.

5. Ren Z.-L., An X.-X., Liu L.-Z., Lan P.-F., Dong W.-K. Synthesis and crystal structure of bis $\left(\mu_{2}\right.$-acetato- $\left.{ }^{2} 0: 0^{\prime}\right)$-di(ethanol)-bis $\left\{\mu_{2}-5\right.$-( $N, N^{\prime}$-diethylamine)-5'-methoxyl-2,2'[ethylenediyldioxybis(nitrilomethylidyne)] diphenolato- ${ }^{6} O$ : 
$O, N, N, O^{\prime}: O^{\prime}$ trinickel(II) - ethanol - acetonitrile (1/2/2), $\mathrm{C}_{58} \mathrm{H}_{86} \mathrm{Ni}_{3} \mathrm{~N}_{8} \mathrm{O}_{18}$. Z. Kristallogr. NCS 2018, 233, 495-497.

6. Lin S., Liu S. X., Lin B. Z. Syntheses, crystal structures and magnetic property of the two $\mathrm{Mn}$ (II) 18-azametallacrown-6 complexes. Chin. J. Inorg. Chem. 2002, 18, 1206-1210.

7. Lin S., Liu S. X., Lin B. Z. Synthesis, crystal structure and magnetic properties of a novel iron(III) 18-azametallacrown-6 compound. Inorg. Chim. Acta 2002, 328, 69-73.
8. Lin S., Liu S. X., Chen Z., Lin B. Z., Gao S. Synthesis, structure, and magnetism of a ferric 24-azametallacrown-8 complex. Inorg. Chem. 2004, 43, 2222-2224.

9. Chen X. H., Liu S. X. Syntheses and crystal structures of two nickel complexes with $\mathrm{N}$-substituted-salicyl ligand. Chin. J. Inorg. Chem. 2005, 21, 15-20.

10. Xiao F.-P., Jin L.-F. Synthesis and characterization of two trinuclear nickel(II) complexes. Z. Anorg. Allg. Chem. 2008, 634, 397-400. 